\title{
Corticosteroid Injection by Palpation Guide versus Palpation Guide and Needling Method for Coccydynia
}

\author{
Ebrahim Ghayem Hassankhani ${ }^{*}$, Golnaz Ghayem Hassankhani², Solmaz Ghayem Hassankhani ${ }^{3}$ \\ ${ }^{1}$ Orthopedic Research Center, Orthopedic Department, Imam Reza Hospital, Mashhad University of Medical Sciences, Mashhad, \\ Iran \\ ${ }^{2}$ Orthopedic Research Center, Ghaem Hospital, Mashhad University of Medical Sciences, Mashhad, Iran \\ ${ }^{3}$ Umeå University, Umeå, Sweden \\ Email: golnazhasankhani@yahoo.com, solmazhasankhani@yahoo.com, ^hasankhanie@mums.ac.ir, *eghasankhani@yahoo.com
}

How to cite this paper: Hassankhani, E.G., Hassankhani, G.G. and Hassankhani, S.G. (2021) Corticosteroid Injection by Palpation Guide versus Palpation Guide and Needling Method for Coccydynia. Open Journal of Orthopedics, 11, 249-256.

https://doi.org/10.4236/ojo.2021.119023

Received: August 1, 2021

Accepted: September 3, 2021

Published: September 10, 2021

Copyright (c) 2021 by author(s) and Scientific Research Publishing Inc. This work is licensed under the Creative Commons Attribution International License (CC BY 4.0).

http://creativecommons.org/licenses/by/4.0/

\begin{abstract}
Background: Coccydynia is a painful condition with tenderness and ache in sacrococcygeal area which may radiate to the buttocks and lower back. Coccydynia is a multifactorial disorder and the most common cause of it is trauma. The initial treatment of the disease is conservative methods. One of the most common conservative treatments of chronic coccydynia is the local corticosteroid injection, which performed usually by palpation-guided method. This study was conducted to compare the effectiveness of local corticosteroid injection using palpation and needling method with palpation method alone. Patients and Methods: In this prospective study, 50 patients with the diagnosis of coccydynia were enrolled between 2010 and 2017. All patients had chronic coccydynia which did not respond to conservative therapy for at least three months. Participants were divided into two groups and each group consisted of 25 individuals. Patients who underwent local corticosteroid injection using palpation guide technique alone were assigned as group $\mathrm{A}$ and others who underwent palpation guiding injection with needling technique were considered as group B. Results: There was a statistically significant difference between the mean VAS scores in preinjection and final follow up visit in each groups ( $\mathrm{P}$ value $<0.001)$. Moreover, the difference between the mean VAS scores of the final visit in groups A and B was statistically significant ( $\mathrm{P}$ value $<0.001$ ). Conclusion: Local corticosteroid injection using palpation guiding technique with needling is an uncomplicated, inexpensive, and effective invasive conservative treatment for refractory chronic coccydynia.
\end{abstract}

\section{Keywords}

Corticosteroid Injection, Palpation Guiding, Needling, Coccydynia 


\section{Background}

Coccydynia or coccygodynia, is an inflammatory condition presents with pain in sacrococcygeal area which may radiate to the buttocks and lower back. Etiology of coccydynia is known to be either local factors or idiopathic, and also it can be a referral pain from other regions [1] [2] [3].

Local factors including traumas (fractures, fracture-dislocation, sprain and child birth), congenital disorders (changes of normal curve or coccygeal configuration), tumors (the most common one is cordoma), degenerative conditions (arthrosis of sacrococcygeal joint), coccygeal intervertebral disc pathology [1] [2], pericoccygeal soft tissue inflammation [2], sacrococcygeal corneal junction pathology [3] and coccygeal nerve entrapment [4].

Unknown Referral pain from other origins such as: lumbosacral injuries and episacral lipoma can be the cause of coccydynia [5] [6] [7].

Although coccydynia is a multifactorial disorder, the most common cause of it is trauma as a result of falling on the buttocks, repetitive micro trauma, or childbirth [6] [8] [9] [10].

Coccygeal configurations are classified into type I, II, III, and IV. Coccyx is gently curved forward in type I, markedly curved forward with the apex directed anteriorly in type II, sharply angulated in type III, and subluxed type IV [7] [11].

The coccyx is a very mobile bone due to its muscle insertions. Also, it serves as the attachment site for the anterior and posterior sacrococcygeal ligaments, the anococcygeal ligaments, as well as the levator ani muscle. Its daily movement aggravates pain in a patient suffering from coccydynia, especially by defecation or sitting [7].

Coccydynia is five times more common in women than men, because the coccyx is more prominent in women. It's presumably more prone to injury [4]-[12]. Also, ligamentous hyperlaxity, obesity [6] [7] and adolescents are other predisposing factors responsible for developing this disease [12] [13].

The diagnosis of coccydynia is made based on history, clinical symptoms and physical examination. Pain of coccyx is typically provoked by sitting [6]. Palpation of coccyx may reveal tenderness and hyper mobility. The pain can be identified by rectal examination [7]. Imaging studies including plain radiography, CTS and MRI, are necessary to identify the shape of coccyx and to exclude the presence of fractures, tumoral or infectious lesions and other pathologies in sacrococcyx region [14].

The initial treatment of the disease is conservative methods, including medications such as non-steroidal anti-inflammatory drugs (NSAIDs), hot baths, ring-shaped cushions, intrarectal massage or manipulation, local injections, ganglion impar blocks, radiofrequency thermo coagulation (RFT), and psychotherapy [4] [14] [15]. Coccygectomy is often recommended if the patient does not respond to conservative treatment [15] [16] [17].

One of the most common treatments of chronic coccydynia is the local corticosteroid injection, which is performed by palpation-guided method. So, this 
study was conducted to compare the effectiveness of local corticosteroids injection using palpation and needling method with palpation method alone.

\section{Patients and Methods}

In this prospective study 50 patients with the diagnosis of coccydynia were enrolled in our study between 2010 and 2017. All patients were treated conservatively with medication, hot baths, ring-shaped cushions, and physiotherapy for at least three months before referring to our orthopedic clinic. The diagnosis of the disease was made through physical examination, laboratory tests, and imaging studies.

The inclusion criteria were as follow for both groups: patients with chronic coccydynia which did not respond to conservative therapy for at least three months. Moreover, the exclusion criteria were patients with contraindications for corticosteroid, previous corticosteroid injection, coccydynia secondary to infection or other bone lesion such as tumor.

In our study patients were divided into two groups and each group consisted of 25 individuals. Patients who underwent local corticosteroid injection using palpation guide technique alone were assigned as group A and other patients who underwent palpation guide injection with needling technique were considered as group B.

\section{Injection Technique}

First, the maximum tenderness point on the coccyx was defined and then lidocaine $2 \%$ was injected to anesthetize the specified tender point in both groups. After anesthetizing the tender point, Depo-Medrol is injected in tender point in group A, but in group B Depo-Medrol injected by moving needle subcutaneously with 1 to $2 \mathrm{~mm}$ intervals in multiple tenderness points. Preferred injection points are: three injections in hyper mobile or tender point of the dorsal surface of the coccyx and one in either lateral borders of the coccyx.

The patients were followed up two, four and six weeks in addition to three and six months after injection therapy. The severity of pain was recorded according to a 10-point visual analogue scale (VAS) before and after injections at all of follow up visits. VAS measures pain intensity subjectively using 10 millimeters line ranging from 0 (no pain) to 10 (very severe pain).

The cost of both methods was almost the same, and the difference was only the price of Lidocaine $2 \%$.

\section{Statistical Analysis}

Statistical analysis was performed using SPSS version 16.0 (SPSS Inc., Chicago IL). Also, a $P$ value of less than 0.05 was considered as statistically significant.

\section{Results}

Demographic characteristics of patients are summarized in Table 1. In our 
Table 1. Demographic characteristics of patients with coccydynia (Total number of patients: 50).

\begin{tabular}{|c|c|c|}
\hline Age (Mean, SD) & \multicolumn{2}{|c|}{$35(9.0)$} \\
\hline Weight (Mean, SD) & \multicolumn{2}{|c|}{$75(6.6)$} \\
\hline \multirow{2}{*}{ Gender $(N, \%)$} & Male & $14(28 \%)$ \\
\hline & Female & $36(72 \%)$ \\
\hline \multirow{6}{*}{ Occupation (N, \%) } & Housewife & $14(28 \%)$ \\
\hline & Employee & $19(38 \%)$ \\
\hline & Driver & $4(8 \%)$ \\
\hline & Worker & $5(10 \%)$ \\
\hline & Student & $6(12 \%)$ \\
\hline & Farmer & $2(4 \%)$ \\
\hline \multirow{3}{*}{ Cause of injury (N, \%) } & Labor & $3.0(6 \%)$ \\
\hline & Trauma & $18(36 \%)$ \\
\hline & Idiopathic & $29(58 \%)$ \\
\hline \multirow{4}{*}{$\mathrm{X}$-Ray report $(\mathrm{N}, \%)$} & Normal & $20(40 \%)$ \\
\hline & Straight & $6.0(12 \%)$ \\
\hline & Angular & $21(42 \%)$ \\
\hline & Subluxation & $3.0(6.0 \%)$ \\
\hline \multirow{2}{*}{ Laxity $(\mathrm{N}, \%)$} & Negative & $16(32 \%)$ \\
\hline & Positive & $34(64 \%)$ \\
\hline \multirow{2}{*}{ Type of injection $(\mathrm{N}, \%)$} & Simple & $25(50 \%)$ \\
\hline & Needling & $25(50 \%)$ \\
\hline Duration of pain (Month) (Mean, SD) & \multicolumn{2}{|c|}{$9.4(2.6)$} \\
\hline VAS score & \multicolumn{2}{|c|}{$8.85(0.83)$} \\
\hline Duration of conservative treatment (Month) (Mean, SD) & \multicolumn{2}{|c|}{$7.8(2.2)$} \\
\hline
\end{tabular}

study, the number of females outweighed the number of males. The mean age of the patients was 35 (SD: 9.0) years ranging from 17 to 54 years. The mean follow up time of the patients were 2.3 (4-1Ys).

Furthermore the etiology of coccydynia in 29 (58\%) patients were unknown (idiopathic), in 18 (36\%) patients were trauma and in $3(6 \%)$ patients were labor. Also, 34 (64\%) patients had laxity of ligaments.

Radiography revealed that shape of the coccyx in majority number of the patients were angular deformity. 21 patients (42\%) were angular, $20(40 \%)$ were normal, 6 (14\%) were straight, and $3(6 \%)$ were subluxed.

The mean duration of the pain in our patients was calculated as 9.4 months, and the mean duration of conservative treatment was 7.8 months (Table 1).

Analysis of VAS scores were shown in Table 2.

In group A (local corticosteroids injection using palpation) the mean pre and post injection (after 6 months or final visit) VAS scores in group A were 8.8 and 3.3 respectively. 
Table 2. Comparing VAS score between simple and needling methods.

\begin{tabular}{ccccc}
\hline & & Simple (Mean, SD) & Needling (Mean, SD) & P-value \\
\hline \multirow{2}{*}{ VAS } & Before treatment & $8.8(0.9)$ & $8.9(0.8)$ & - \\
& After treatment & $3.3(0.6)$ & $1.4(0.5)$ & $<0.001$ \\
& P-value & $<0.001$ & $<0.001$ & \\
\hline
\end{tabular}

Also the mean VAS scores in group B were 8.9 preinjection, and 1.4 after six months (final visit).

Our analysis showed that there was a statistically significant difference between the mean VAS scores in preinjection and final follow up visit in each groups ( $\mathrm{P}$ value $<0.001$ ).

Moreover the difference between the mean VAS scores of the final visit in groups A (3.3) and B (1.4) were statistically significant $(\mathrm{P}$ value $<0.001)$ (Table 2).

\section{Discussion}

In this study, we compared the efficacy of two corticosteroid injection techniques (local corticosteroid injection using palpation alone method versus palpation and needling method) in treatment of coccydynia. Our study revealed a significant difference considering the mean VAS scores between preinjection and final follow up visit either within a group or between two groups.

It has been reported that the etiology of the coccydynia is multifactorial. In between the most commonly reported etiology is trauma, but in our study the majority of cases was idiopathic coccydynia (58\%) [1] [4] [5] [8] [9] [10] [18] [19].

Furthermore, coccygeal configuration is another etiological factor, which is identified using lateral radiography [5] [7] [11] [22].

Although, many authors believe that patients with coccydynia are more likely to have a coccygeal configuration other than type I [5] [7], our study showed that the prevalence of type I and type III were $40 \%$ and $42 \%$ respectively.

Also, $64 \%$ of our patients had hyper mobile coccyx which is another important factor in presence of the disease due to chronic inflammatory changes [11].

In terms of prevalence, our study confirmed the fact that the incidence of this disease is higher among females [20] [21].

Conservative managements of coccydynia are the gold standard treatments, consisting of medications such as NSAIDs and analgesics, reduced sitting, hot baths, ring shape pillow or Circular cushions (donut cushions), adopting proper sitting posture and physical therapy [12] [21] [22].

Although conservative treatments are successful in $90 \%$ of cases, many resolves are without any interventions [12] [23]. If the patients do not respond to non-invasive conservative therapies after 2 months, diagnosed chronic coccygodynia and should receive invasive measures [21].

One of the invasive therapeutic approaches is local corticosteroid injections 
into the coccyx region which can be repeated up to three times if a patient has persistent symptoms [22].

In this study, local corticosteroid injection was done in cases who did not respond to non-invasive conservative treatments for at list three months and there was not any case for injection repeat.

In all literatures the method of local corticosteroid injection are simple as our group A, and a few literatures report injection in three point (dorsal and lateral) like our group B. In our new method we did at list five injections in three points but subcutonaneously with one injection (three in hyper mobile and tender point of dorsal of coccyx and one in each lateral border of coccyx).

Considering the fact that limited studies investigated the benefits of local corticosteroid injection, acceptable post injection results have been reported According to VAS scores [19] [24].

There is a study that demonstrated a significant immediate pain relief after a Fluoroscopically Guided coccygeal steroid injection [19]. Although we used blind local corticosteroid injection just by detecting a tender point, we found similar results. Another study compared the results of local injection and manipulation in contrast to local injection alone. This study reported that post injection coccygeal manipulation was more advantageous than injection alone; it was found that patients slightly better when they received post injection manipulation (59\% vs. $85 \%)$ [20].

We found that pain in both groups was significantly decreased after injection. Further, pain relief was notably significant in group B (injection with needling) in contrast to group A (injection alone). So, using corticosteroid injection with needling would be more beneficial as it is simple, cheap, and not dependent to fluoroscopy.

\section{Conclusion}

Local corticosteroid injection using palpation guide technique with needling is an uncomplicated, inexpensive, and effective invasive conservative treatment for coccydynia, mostly in patients with refractory chronic coccydynia who had not been cured after at least three months of non-invasive conservative therapies.

\section{Acknowledgements}

The authors thank orthopedic research center group of Mashhad Medical University for their assistance.

\section{Conflicts of Interest}

The authors declare no conflicts of interest regarding the publication of this paper.

\section{References}

[1] Haghighat, S. and Asl, M.M. (2016) Effects of Extracorporeal Shock Wave Therapy 
on Pain in Patients with Chronic Refractory Coccydynia: A Quasi-Experimental Study. Anesthesia and Pain Medicine, 6, e37428. https://doi.org/10.5812/aapm.37428

[2] Woon, J.T., Maigne, J.Y., Perumal, V. and Stringer, M.D. (2013) Magnetic Resonance Imaging Morphology and Morphometry of the Coccyx in Coccydynia. Spine, 38, 1437-1445. https://doi.org/10.1097/BRS.0b013e3182a45e07

[3] Woon, J.T. and Stringer, M.D. (2012) Clinical Anatomy of the Coccyx: A Systematic Review. Clinical Anatomy, 25, 158-167. https://doi.org/10.1002/ca.21216

[4] Nathan, S.T., Fisher, B.E. and Roberts, C.S. (2010) Coccydynia: A Review of Pathoanatomy, Aetiology, Treatment and Outcome. The Journal of Bone and Joint Surgery. British Volume, 92, 1622-1627.

https://doi.org/10.1302/0301-620X.92B12.25486

[5] Howard, P.D., Dolan, A.N. and Falco, A.N. (2013) A Comparison of Conservative Interventions and Their Effectiveness for Coccydynia: A Systematic Review. Journal of Manual and Manipulative Therapy, 21, 213-219. https://doi.org/10.1179/2042618613Y.0000000040

[6] Patijn, J., Janssen, M., Hayek, S., Mekhail, N., Van Zundert, J. and van Kleef, M. (2010) Coccygodynia. Pain Practice, 10, 554-559. https://doi.org/10.1111/j.1533-2500.2010.00404.X

[7] Kerr, E.E., Benson, D. and Schrot, R.J. (2011) Coccygectomy for Chronic Refractory Coccygodynia: Clinical Case Series and Literature Review. Journal of Neurosurgery: Spine, 14, 654-663. https://doi.org/10.3171/2010.12.SPINE10262

[8] Maigne, J., Doursounian, L. and Chatellier, G. (2000) Causes and Mechanisms of Common Coccydynia: Role of Body Mass Index and Coccygeal Trauma. Spine, 25, 3072-3079. https://doi.org/10.1097/00007632-200012010-00015

[9] Emerson, S.S. and Speece, A.J. (2012) Manipulation of the Coccyx with Anesthesia for the Management of Coccydynia. The Journal of the American Osteopathic Association, 112, 805-807.

[10] Fogel, G.R., Cunningham, P.Y. and Esses, S.I. (2004) Coccygodynia: Evaluation and Management. Journal of the American Academy of Orthopaedic Surgeons, 12, 49-54. https://doi.org/10.5435/00124635-200401000-00007

[11] Patel, R., Appannagari, A. and Whang, P.G. (2008) Coccydynia. Current Reviews in Musculoskeletal Medicine, 1, 223-226. https://doi.org/10.1007/s12178-008-9028-1

[12] Lirette, L.S., Chaiban, G. and Tolba, R. (2014) Coccydynia: An Overview of the Anatomy, Etiology, and Treatment of Coccyx Pain. The Ochsner Journal, 14, 84-87.

[13] Maigne, J.Y., Pigeau, I., Aguer, N., Doursounian, L. and Chatellier, G. (2014) Chronic Coccydynia in Adolescents. A Series of 53 Patients. European Journal of Physical and Rehabilitation Medicine, 47, 245-251.

[14] Karadimas, E.J., Trypsiannis, G. and Giannoudis, P.V. (2011) Surgical Treatment of Coccygodynia: An Analytic Review of the Literature. European Spine Journal, 20, 698-705. https://doi.org/10.1007/s00586-010-1617-1

[15] Lirette, L.S., Chaiban, G., Tolba, R. and Eissa, H. (2014) Coccydynia: An Overview of the Anatomy, Etiology, and Treatment of Coccyx Pain. Ochsner Journal, 14, 84-87.

[16] Haddad, B., Prasad, V., Khan, W., Alam, M. and Tucker, S. (2014) Favourable Outcomes of Coccygectomy for Refractory Coccygodynia. Annals of the Royal College of Surgeons of England, 96, 136-139. https://doi.org/10.1308/003588414X13814021676675 
[17] Antoniadis, A., Ulrich, N.H. and Senyurt, H. (2014) Coccygectomy as a Surgical Option in the Treatment of Chronic Traumatic Coccygodynia: A Single Center Experience and Literature Review. Asian Spine Journal, 8, 705-710. https://doi.org/10.4184/asj.2014.8.6.705

[18] Maigne, J.Y., Rusakiewicz, F. and Diouf, M. (2012) Postpartum Coccydynia: A Case Series Study of 57 Women. European Journal of Physical and Rehabilitation Medicine, 48, 387-392.

[19] Mitra and Cheung (2007) Retrospective Evaluation Efficacy of Fluoroscopically Guided Steroid Injections in the Management of Coccydynia. Pain Physician, 10, 775-778. https://doi.org/10.36076/ppj.2007/10/775

[20] Wray, C.C., Easom, S.S. and Hoskinson, J. (1991) Coccydynia: Etiology and Treatment. The Journal of Bone and Joint Surgery. British Volume, 73, 335-338. https://doi.org/10.1302/0301-620X.73B2.2005168

[21] Kwon, H.D., Schrot, R.J. and Kerr, E.E. (2012) Coccygodynia and Coccygectomy. Korean Journal of Spine, 9, 326-333. https://doi.org/10.14245/kjs.2012.9.4.326

[22] Patel, R., Appannagari, A. and Whang, P.G. (2008) Coccydynia. Current Reviews in Musculoskeletal Medicine, 1, 223-226. https://doi.org/10.1007/s12178-008-9028-1

[23] Trollegaard, A.M., Aarby, N.S. and Hellberg, S. (2010) Coccygectomy: An Effective Treatment Option for Chronic Coccydynia: Retrospective Results in 41 Consecutive Patients. The Journal of Bone and Joint Surgery. British Volume, 92, 242-245. https://doi.org/10.1302/0301-620X.92B2.23030

[24] Perkins, R., Schofferman, J. and Reynolds, J. (2003) Coccygectomy for Severe Refractory Sacrococcygeal Joint Pain. Journal of Spinal Disorders and Techniques, 16, 100-103. https://doi.org/10.1097/00024720-200302000-00016 